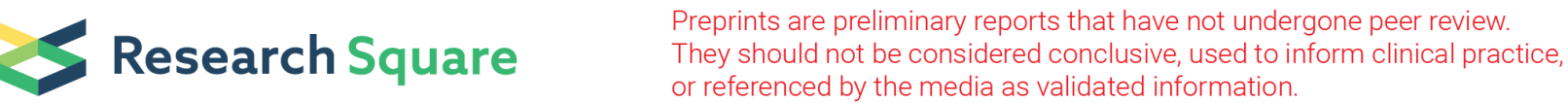

\section{Optimal Extent of Lymphadenectomy for Radical Surgery of Pancreatic Head Adenocarcinoma: A Single-center, Prospective, Randomized Controlled Study}

\section{Ziyao Wang}

Department of Pancreatic Surgery, West China Hospital, Sichuan University

\section{Xin Wang}

Sichuan University West China Hospital Department of Pancreatic Surgery

\section{Xing Wang}

Sichuan University West China Hospital of Pancreatic Surgery

\section{Yonghua Chen}

Sichuan University West China Hospital

Jinheng Liu

Sichuan University West China Hospiatal of Pancreatic Surgery

Du He

Department of Pathology, West China Hospital, Sichuan University

\section{Bole Tian}

Department of Pancreatic Surgery, West China Hospital, Sichuan University

\section{Ang Li}

Department of Pancreatic Surgery, West China Hospital,Sichuan University

\section{Weiming Hu}

Department of Pancreatic Surgery, West China Hospital, Sichuan University

\section{Kezhou Li}

Department of Pancreatic Surgery, West China Hospital, Sichuan University

\section{Nengwen Ke}

Department of Pancreatic Surgery, West China Hospital, Sichuan University

Xubao Liu ( $\nabla$ xbliu@medmail.com.cn )

Sichuan University

\section{Research article}

Keywords: pancreatic adenocarcinoma, lymph node dissection, extent, survival analysis

Posted Date: November 5th, 2020 
DOl: https://doi.org/10.21203/rs.3.rs-101464/v1

License: (c) (1) This work is licensed under a Creative Commons Attribution 4.0 International License. Read Full License 


\section{Abstract}

\section{Background}

Radical pancreaticoduodenectomy is the only possible cure for pancreatic head adenocarcinoma, and although several RCT studies have suggested the extent of lymph node dissection, this issue remains controversial. This article wanted to evaluate the survival benefit of different lymph node dissection extent for radical surgical treatment of pancreatic head adenocarcinoma.

Methods

A total of 240 patients were enrolled in the study, 212 of whom were randomly divided into standard lymphadenectomy group (SG) or extended lymphadenectomy group (EG), there were 97 patients in SG and 95 patients in EG receiving the radical pancreaticoduodenectomy.

Result

The demography, histopathology and clinical characteristics were similar between the two groups. The 2year overall survival rate in the SG was higher than the EG (39.5\% vs $25.3 \% ; p=0.034)$. The 2-year overall survival rate in the SG who received postoperative adjuvant chemotherapy was higher than the EG $(60.7 \%$ vs $37.1 \% ; p=0.021)$. There was no significant difference in the overall incidence of complications between the two groups ( $p=0.502)$. The number of peripheral blood lymphocytes of the EG was significantly lower than that in the SG at 1 week (mean [SD], 0.957[0.429] vs 1.278[0.521], $p=0.001$ ) and 1 month (mean [SD], $1.538[0.618]$ vs $1.917[0.796], p=.009)$ after operation.

\section{Conclusion}

In multimodality therapy system, the efficacy of chemotherapy should be based on the appropriate lymphadenectomy extent, and the standard extent of lymphadenectomy is optimal for resectable pancreatic head adenocarcinoma. The postoperative slowing of peripheral blood lymphocyte recovery might be one of the reasons why extended lymphadenectomy did not result in survival benefits.

Clinical trial registration

This trial was registered at ClinicalTrials.gov (NCT02928081) in October 7, 2016. https://clinicaltrials.gov/

\section{Background}

Pancreatic cancer is one of the most lethal diseases of the digestive system.[1] By 2030, pancreatic cancers are projected to surpass breast, prostate, and colorectal cancers to become the second leading cause of cancer-related death.[2] Presently, surgical resection, in combination with systemic 
chemotherapy, offers the only hope of cure for patients with pancreatic cancer.[3] Therefore, how to improve surgical outcomes is a crucial aim of current research.

Since the 1980s, Japanese researchers have recommended retroperitoneal lymphadenectomy in pancreatoduodenectomy (PD).[4] Since then, it has become popular to assert that extended lymphadenectomy in pancreatoduodenectomy (EPD) is superior to standard lymphadenectomy in pancreatoduodenectomy (SPD). However, subsequent studies have found that EPD does not result in survival benefits and might increase the incidence of postoperative complications.[5-10] In 2014, the International Pancreatic Surgery Research Group (ISGPS) reached a consensus about SPD that includes the $5,6,8 a, 12 b 1,12 b 2,12 c, 13 a, 13 b, 14 a, 14 b, 17 a$, and 17b lymph nodes.[11] Furthermore, some researchers reported that lymphadenectomy that includes the 12b,12c, 13, and 17 lymph nodes can be performed safely and efficiently, without negatively affecting oncologic efficacy or long-term survival, only compared with EPD.[9] Therefore, at present, the extent of lymphadenectomy in PD remains controversial.

We designed this randomized controlled study based on the 2014 consensus of the ISGPS. To determine the optimal lymphadenectomy extent for PD in the treatment of pancreatic head adenocarcinoma, we studied the effect of SPD and EPD on the survival time of patients.

\section{Materials And Methods}

\section{Trial Design}

This randomized, controlled, single-center, single-blind (subject), parallel-group trial compared standard lymphadenectomy versus extended lymphadenectomy in PD for treatment of pancreatic head adenocarcinoma. It complied with the Declaration of Helsinki, was approved and overseen by the institutional review board of West China Hospital, and is registered at ClinicalTrials.gov (NCT02928081).

\section{Participants}

All patients enrolled in this study were treated in the pancreatic surgery department of West China Hospital from October 2016 to May 2018, and all operations were performed by seven different surgeons who specialize in pancreatic surgery. Inclusion criteria were age below 75 , pancreatic head ductal adenocarcinoma, resectable (NCCN Guidelines Pancreatic Adenocarcinoma Version 2.2016), no history of tumor. Exclusion criteria were unresectable or metastasis, with serious diseases of other organs (e.g., coronary heart disease), and other pathology. All patients had to sign informed consent forms before inclusion in the study. Patients selected for analysis from prospective database used the same criteria described above. Data were analyzed in May 2020.

Interventions

According to the 2014 ISGPS consensus statement, in SPD, the resected lymph nodes included 5, 6, 8a, 12b, 12c, 13, 14a, 14b, and 17. In EPD, in addition to those lymph nodes removed in SPD, the following 
nodes were resected as well: $9,12 p, 14 c, 14 d, 16 a 2$, and 16b1, and all soft tissues surrounding the hepatoduodenal ligament were dissected and skeletonized, and the right celiac plexus and superior mesenteric artery right plexus were resected. The differences in specific resection extent are shown in Supplement Table 1. We recommended postoperative chemotherapy to all patients, except for patients with poor physical condition or organ insufficiency, or those who refused adjuvant chemotherapy. All patients underwent surgical field photographs to verify that the required surgical extent was achieved. All photos and data are monitored and stored by the clinical trial center of West China Hospital of Sichuan University. The chemotherapy regimen included gemcitabine (1000 mg per square meter on days 1, 8, and 15 every 4 weeks) for 24 weeks.

\section{Outcomes}

The primary endpoint of this study was 2-year overall survival (OS), which was defined as the time from randomization to death. The secondary endpoints were morbidity and postoperative mortality.

Sample size

Our trial was powered for superiority of survival data at 2 years according to the previous trial, assuming that the 2-year survival rate (38\%) of patients who underwent extended pancreatectomy was $18 \%$ higher than that of the standard group (20\%).[12, 13] [14]With alpha $=0.05$, beta $=0.20$, and the power of $90 \%$, assuming a drop-out rate of $20 \%$, a total sample size of $n=200$ patients would be allocated to this trial and a sample size of 100 per group is necessary to detect a difference between the intervention groups, because of the high dropout rate $(>20 \%)$, we added an additional 30 patients during the recruitment, using PASS 11.0 software (NCSS, LLC, Kaysville, Utah).

\section{Randomization}

After confirming patient eligibility and determining that the tumor could be removed, patients were randomly assigned 1:1 using the Multi Random Data Generator. Sealed and numbered envelopes that contained the allocated group were prepared and opened before surgery.

Statistical Analysis

Patient eligibility for follow-up and analysis was determined by evaluating the photographs taken during the operation. The results are presented as mean \pm standard error or median with interquartile range. Nominal data were compared using the Chi-squared test, and continuous variables were analyzed with Student's t-test. The Kruskal-Wallis test was used for data that did not conform to the normal distribution. Survival data were calculated using the Kaplan-Meier method and compared using the logrank test. A Cox proportional hazards regression model was used to calculate the effect of multiple factors on survival time, and variables that presented significant differences in univariate analysis were included in the final analysis. All statistical analyses were performed using SPSS 23.0 software (SPSS Inc, Chicago, Illinois), and two-sided $P$ values less than 0.05 were considered statistically significant. 


\section{Results}

\section{Demographics}

A total of 240 patients were enrolled in this randomized controlled study. Of these, 11 patients were excluded because of declined to participate (Figure. 1). The remaining 212 patients were randomly divided 1:1 into the SPD group (SG) or EPD group (EG). In total, we analyzed 153 patients, including 79 who underwent SPD and 74 who underwent EPD. The inclusion and exclusion criteria for this group of patients were consistent with the aforementioned criteria. Therefore, the patients in two groups matched well in terms of age, gender, nutritional status, preoperative conditions, portal vein resection rate, tumor staging, and follow-up time (Table 1). The number of patients who received postoperative adjuvant chemotherapy was 32 in SG, and 29 in EG.

\section{Pathologic Differences}

Table 1 shows the pathologic differences among the three groups. No significant differences were observed in the $\mathrm{R} 1$ resection rate among the two group $(P=0.768)$, and no significant differences were observed among the two groups in tumor size $(P=0.870)$ and T stage $(P=0.790)$, or American Joint Committee on Cancer (8th edition) stage $(P=0.733)$. In terms of retrieving the number of lymph nodes, the EG was significantly higher than the SG (24 [range, 22-26] vs 18 [range, 16-19]; $P<0.001)$.

Morbidity and Mortality

No significant differences were observed in morbidity and mortality between the SG and EG $(39.2 \%$ vs $44.6 \%, P=0.502$ ) (Table 2). Because two patients in the SG had long postoperative hospital stays ( $>60$ days), the average postoperative hospital stay in this group was slightly longer than the average postoperative hospital stay in the EG, but the difference was not significant (mean [SD] days 14.66 [10.32] vs 12.65 [4.93], $P=0.131)$. Three patients in the SG (3.8\%) died, one of postoperative intraabdominal bleeding, the others of respiratory failure due to sepsis and pulmonary infection. Two patients in the EG (2.7\%) died, both of intra-abdominal bleeding.

\section{Survival Data}

After excluding deaths in the hospital, survival analysis was performed on 75 patients in the SG, on 71 patients in the EG. The median survival time of the enrolled patients was 22 months (SG), and 15 months (EG). The 2-year OS rate were 39.5\% (SG), and 25.3\% (EG). The 2-year OS rate of patients in the SG was higher than that of patients in the EG $(P=0.034)$ (Figure $2 A)$. The 2-year DFS rate of patients in the SG was higher than that of patients in the $E G(28.25 \%$ vs $19.32 \% ; \mathrm{P}=0.046)$ (Figure $2 \mathrm{~B})$. For intention-to-treat analysis, which included all patients who finally received the radical pancreaticoduodenectomy randomly assigned to the standard ( $n=97)$ and extended $(n=95)$ groups, the 2-year OS rate was $41.0 \%$ and $26.2 \%$ $(\mathrm{P}=0.023)$, respectively (Figure $2 \mathrm{C})$. 
The 2-year OS rate of patients who received postoperative chemotherapy was higher than that of patients who did not receive chemotherapy ( $S G, P<0.001 ; E G, P=0.009)$ (Supplement Figure 1). The 2-year OS rate of patients in the SG who received postoperative chemotherapy

was higher than that of patients in the $E G(60.7 \%$ vs $37.1 \% ; P=0.021)$ (Figure $3 \mathrm{~A})$. Patients who did not receive chemotherapy showed no difference in 2-year OS rate between the SG and EG (31.6\% vs 20.2\%; $P$ $=0.366$ ) (Figure 3B). No differences were observed in the survival rate of lymph node-positive patients between the SG and EG (28.6\% vs $18.1 \%$; $P=0.065)$ (Figure 3C). Although the 2-year OS rate of lymph node-negative patients in the SG was higher than that of lymph node-negative patients in the EG, no significant differences were observed between two groups (55.3\% vs 35.2\%; $P=0.182$ ) (Figure 3D).

For patients with positive lymph nodes who received postoperative chemotherapy, no differences were observed in the 2-year OS rate between the SG and EG (37.5\% vs $49.0 \% ; \mathrm{P}=0.698$ ) (Figure 3E). However, for patients with negative lymph nodes who received postoperative chemotherapy, the 2-year OS rate of patients in the SG was higher than that of patients in the EG (87.5\% vs $30 \% ; \mathrm{P}=0.004)$ (Figure 3F).

In addition, for patients with negative lymph nodes who did not receive postoperative chemotherapy, no differences were observed in the 2-year OS rate between the SG and EG ( $26.5 \%$ vs $40.8 \% ; P=0.491$ ) (Figure $3 \mathrm{H}$ ). However, for patients with positive lymph nodes who did not receive postoperative chemotherapy, the 2-year OS rate of patients in the SG was higher than that of patients in the EG (23.0\% vs 7.1\%; $P=0.036$ ) (Figure 3G).

\section{Prognostic Factors}

Univariate analysis showed that the insufficient extent of surgical dissection, regional lymph node metastasis, later stage, poor histologic differentiation, and absence of postoperative adjuvant chemotherapy were associated with adverse outcomes (Table 3). In the multivariate Cox proportional hazards model, lymphadenectomy extent (hazard ratio [HR] = 1.67; 95\% confidence interval [CI], 1.1302.254; $P=0.010)$, Stage $(H R=1.338 ; 95 \% \mathrm{Cl}, 0.992-1.804 ; P=0.056)$, histologic differentiation $(H R=$ $2.190 ; 95 \% \mathrm{Cl}, 1.370-3.502 ; \mathrm{P}=0.001)$, and postoperative adjuvant chemotherapy $(\mathrm{HR}=2.226 ; 95 \% \mathrm{Cl}$, $1.464-3.384 ; \mathrm{P}<0.001)$ remained statistically significant.

The peripheral blood lymphocyte levels of all patients who underwent PD experienced a process of declining and then slowly rising during the perioperative period. However, the lymphocyte count of patients in the EG was significantly lower than the lymphocyte count of patients in the SG (mean [SD] $\times 10^{9}, 0.957$ [0.429] vs 1.278 [0.521]; $\left.P=0.001\right)$ at 1 week, and the lymphocyte count of patients in the EG was significantly lower than the lymphocyte count of patients in the SG (mean [SD] $\times 10^{9}, 1.538[0.618]$ vs 1.917 [0.796]; $P=0.009$ ) at 1 month (Figure 2D).

\section{Discussion}


Previously, Pedrazzoli et al. suggested that extended lymphadenectomy can be performed in some patients, and patients with positive lymph nodes seem to benefit from extended dissection.[5] However, a subsequent randomized controlled study by Yeo et al. showed that the 5-year OS rate and median survival time of patients with negative lymph nodes were significantly higher than patients with positive lymph nodes, this conclusions possibly because approximately $78 \%$ of the patients in this study received different types of postoperative adjuvant treatment.[6] An additional study by Farnell et al. included only patients with adenocarcinoma of the pancreatic head. Their results showed that extended lymphadenectomy did not improve survival time regardless of lymph node status.[7] Although there was some ethical controversy, none of the patients enrolled in a Japanese randomized controlled study received postoperative adjuvant treatment, and the final results were similar to Farnell et al.'s report.[8] Interestingly, patients who received extended lymphadenectomy had a higher local recurrence rate, which, it is speculated, might have been influenced by increased immunologic suppression associated with the more extensive resection.[8] However, this explanation is not supported by relevant data. Subsequently, Korean researchers further confirmed that extended lymphadenectomy did not improve patient survival time compared to standard lymphadenectomy, whether the lymph nodes were positive or negative, and lacking of postoperative adjuvant treatment may be the primary factor influencing prognosis. $[9,10]$

The past five randomized controlled studies had different definitions of standard lymphadenectomy. Because the most commonly metastatic lymph nodes of pancreatic head cancer were No. 14, which have an important effect on the prognosis,[15] standard lymphadenectomy that includes No. 14 lymphadenectomy has gradually become an international consensus.[11] Therefore, the Japanese and Korean studies might have ignored the potential survival benefits of No.14 lymphadenectomy.

Our survival data showed that the 2-year OS and DFS rate of patients in the SG were better than the 2year OS and DFS rate of patients in the EG. This is different from the results of previous randomized controlled studies, and the most important reason is the different definition of the standard. The standard extent of lymphadenectomy performed by Farnell et al. was similar to ours[7], but their results did not indicate that the survival time of the standard group was superior to the survival time of the extended group. We speculate that the primary reason for this discrepancy is their small sample size.[7] Some studies found that the postoperative peripheral blood lymphocytes of patients who underwent PD experienced a polyline change (sharp decline, slow rise, and gradual recovery).[16, 17] Patients who do not fully and rapidly recover the level of lymphocytes after the operation are considered to be prone to recurrence and have a poor prognosis.[16] We measured the trend of lymphocytes after operation in two groups of patients, the slowdown of lymphocyte recovery of patients in the extended resection group may be the reason why extended lymphadenectomy will contrarily bring higher local recurrence rate and worse survival time. This suggests that the impact of surgical stress on the immune system is likely to affect the recurrence rate and survival rate.

Even though chemotherapy after radical resection can significantly improve survival rate, and modified FOLFIRINOX regimen led to significantly longer disease-free survival and overall survival than adjuvant chemotherapy with gemcitabine, but most our patients cannot tolerate the modified FOLFIRINOX regimen, 
Which led to the lower overall survival rate we reported to compare with that reported by Conroy et al.[18] Subgroup analysis revealed that postoperative chemotherapy could significantly improve the survival time of patients regardless of extent of lymphadenectomy, and the 2-year OS rate of patients in the SG who received postoperative chemotherapy was significantly higher than the 2-year OS of patients in the EG $(60.7 \%$ vs $37.1 \% ; P=0.021)$, which might be related to the recovery of patients' immune systems. The above analysis suggests that the efficacy of chemotherapy should be based on the appropriate extent of lymphadenectomy, while extended lymphadenectomy (including 16a2 and 16b1) is likely to lead to higher local recurrence rate and poor efficacy of chemotherapy. Further analysis showed that the patients in the SG with negative lymph nodes who received postoperative chemotherapy had the highest survival rate because of the effective local radical lymphadenectomy and reasonable systemic therapy. However, this advantage did not exist in patients in the SG with positive lymph nodes who received postoperative chemotherapy, supporting an approach that minimizes the extent of lymphadenectomy to lymph nodes that have a clear histologic metastasis to avoid the excessive surgical trauma and ensuring the effect of chemotherapy. Thus, the studies of Nimura et al. and Jang et al. adopted a limited "standard" lymphadenectomy.[8-10] Some of our patients also received the "standard" extent of lymphadenectomy \only No.12b,12c,13 and 17区 reported by Jang et al, survival analysis of this part of patients showed that their 2-year survival rate was not statistically different from the EG group $(26.1 \%$ vs $25.3 \% ; P=0.484)$ (Supplement Figure 2B), but was lower than the SG group (26.1\% vs 39.5; $P=0.047$ ) (Supplement Figure $2 A)$, which means that although a limited extent of lymphadenectomy \only No.12b,12c,13 and 17凶can obtain a similar survival time as the EG group, it may also lose the survival benefits of dissection of the N0.14 lymph nodes. Of course, this result was only based on the analysis of current survival data and has not been confirmed by RCT trials.

Early pancreatic cancer is considered a systemic disease because the formation of local and regional micrometastases is inevitable.[19] Through a reasonable lymphadenectomy (No. 14), patients with a relatively normal immune system who receive postoperative chemotherapy can survive and benefit. According to the Cox proportional hazards model, the most important factors affecting prognosis were the extent of lymphadenectomy, chemotherapy, staging, and differentiation. More extensive lymphadenectomy is not suitable to improve the survival rate in all cases; rather, to increase the survival of patients with pancreatic head cancer, it is reasonable to propose that proper systemic treatment after surgery is better than extended lymphadenectomy. Pancreas includes the fusion of the ventral pancreas and dorsal pancreas. Therefore, some studies have pointed out that, when the tumor is located in the ventral pancreas, lymph node metastasis and nerve invasion often occur around the superior mesenteric artery and pancreatic head nerve plexus.[20, 21] However, if the tumor is located in the dorsal pancreas, lymph node metastasis and nerve invasion often present in the common hepatic artery and hepatoduodenal ligament region.[20, 21] This means that although extended lymphadenectomy is not recommended in all studies, there might be more individualized options for standard or limited lymph node dissection that could be identified through future randomized controlled studies grouped by different tumor sites. 


\section{Conclusion}

The standard extent of lymphadenectomy is optimal for resectable pancreatic head adenocarcinoma, and the efficacy of chemotherapy should be based on the appropriate extent of lymphadenectomy to improve patient's survival.

\section{Abbreviations}

SG: standard lymphadenectomy group; EG: extended lymphadenectomy group; PD: pancreatoduodenectomy; SPD: standard lymphadenectomy in pancreatoduodenectomy; EPD: extended lymphadenectomy in pancreatoduodenectomy; OS: overall survival

\section{Declarations}

\section{Ethics approval and consent to participate}

Ethical approval for this study (Ethics Committee No. 2016-122) was provided by the Ethical Committee of West China Hospital of Sichuan University on 26 October 2016. Written informed consent was obtained from the study participants.

\section{Consent for publication}

Not applicable

\section{Availability of data and materials}

The datasets used and/or analyzed during the current study are available from the corresponding author on reasonable request.

\section{Competing interests}

The authors declare that they have no competing interests.

\section{Funding}

This study was funded by the West China Hospital, Sichuan University, China (The 1.3.5 Project for Disciplines of Excellence-Clinical Research Incubation Project, Xubao Liu ZY2017302), they financed the cost of the study and contributed to the design of the study, interpretation of data, and writing the manuscript. The Key Research and Development Projects in Sichuan Province, China (Xubao Liu 2019YFS0043, Nengwen Ke 20ZDYF2735) partly financed the cost of the study and contributed to the collection of data and patient follow-up.

\section{Authors' contributions}


$X B L$ and NW K contributed to conception and design, ZY W and X W1 drafted the article and revised it critically for important intellectual content. X W2, YH C, HY C, JH L, D H contributed to acquisition of data and interpretation of data. BL T, WM H, KZ L, A L contributed to surgery. All authors read and approved the final manuscript.

\section{Acknowledgements}

Not applicable.

\section{References}

1. Kang MJ, Jang JY, Kim SW. Surgical resection of pancreatic head cancer: What is the optimal extent of surgery. Cancer Lett. 2016. 382(2): 259-265.

2. Rahib L, Smith BD, Aizenberg R, Rosenzweig AB, Fleshman JM, Matrisian LM. Projecting cancer incidence and deaths to 2030: the unexpected burden of thyroid, liver, and pancreas cancers in the United States. Cancer Res. 2014;74(11):2913-2921.

3. Strobel O, Neoptolemos J, Jäger D, Büchler MW. Optimizing the outcomes of pancreatic cancer surgery. Nat Rev Clin Oncol. 2019;16(1):11-26.

4. Ishikawa $\mathrm{O}$, Oohigashi $\mathrm{H}$, Imaoka S, et al. [Clinico-pathological study on the appropriate range of pancreatic resection to obtain operative curability of pancreatic head cancer]. Nihon Geka Gakkai Zasshi. 1984;85(4):363-369.

5. Pedrazzoli S, DiCarlo V, Dionigi R, et al. Standard versus extended lymphadenectomy associated with pancreatoduodenectomy in the surgical treatment of adenocarcinoma of the head of the pancreas: a multicenter, prospective, randomized study. Lymphadenectomy Study Group. Ann Surg. 1998;228(4):508-517.

6. Yeo CJ, Cameron JL, Lillemoe KD, et al. Pancreaticoduodenectomy with or without distal gastrectomy and extended retroperitoneal lymphadenectomy for periampullary adenocarcinoma, part 2: randomized controlled trial evaluating survival, morbidity, and mortality. Ann Surg. 2002;236(3):355-366; discussion 366-368.

7. Farnell MB, Pearson RK, Sarr MG, et al. A prospective randomized trial comparing standard pancreatoduodenectomy with pancreatoduodenectomy with extended lymphadenectomy in resectable pancreatic head adenocarcinoma. Surgery. 2005;138(4):618-628; discussion 628-630.

8. Nimura Y, Nagino M, Takao S, et al. Standard versus extended lymphadenectomy in radical pancreatoduodenectomy for ductal adenocarcinoma of the head of the pancreas: long-term results of a Japanese multicenter randomized controlled trial. J Hepatobiliary Pancreat Sci. 2012;19(3):230241.

9. Jang JY, Kang MJ, Heo JS, et al. A prospective randomized controlled study comparing outcomes of standard resection and extended resection, including dissection of the nerve plexus and various lymph nodes, in patients with pancreatic head cancer. Ann Surg. 2014;259(4):656-664. 
10. Jang JY, Kang JS, Han Y, et al. Long-term outcomes and recurrence patterns of standard versus extended pancreatectomy for pancreatic head cancer: a multicenter prospective randomized controlled study. J Hepatobiliary Pancreat Sci. 2017;24(7):426-433.

11. Tol JA, Gouma DJ, Bassi C, et al. Definition of a standard lymphadenectomy in surgery for pancreatic ductal adenocarcinoma: a consensus statement by the International Study Group on Pancreatic Surgery (ISGPS). Surgery. 2014;156(3):591-600.

12. Henne-Bruns D, Vogel I, Lüttges J, Klöppel G, Kremer B. Ductal adenocarcinoma of the pancreas head: survival after regional versus extended lymphadenectomy. Hepatogastroenterology. 1998;45(21):855-866.

13. Ishikawa O, Ohhigashi H, Sasaki Y, et al. Practical usefulness of lymphatic and connective tissue clearance for the carcinoma of the pancreas head. Ann Surg. 1988;208(2):215-220.

14. Mukaiya M, Hirata K, Satoh T, Kimura M, Yamashiro K, Ura H, et al. Lack of survival benefit of extended lymph node dissection for ductal adenocarcinoma of the head of the pancreas: retrospective multi-institutional analysis in Japan. World J Surg. 1998;22:248-52; discussion 252-3.

15. Malleo G, Maggino L, Capelli P, et al. Reappraisal of Nodal Staging and Study of Lymph Node Station Involvement in Pancreaticoduodenectomy with the Standard International Study Group of Pancreatic Surgery Definition of Lymphadenectomy for Cancer. J Am Coll Surg. 2015;221(2):367-379.e4.

16. Kim EY, Hong TH. Changes in total lymphocyte count and neutrophil-to-lymphocyte ratio after curative pancreatectomy in patients with pancreas adenocarcinoma and their prognostic role. J Surg Oncol. 2019;120(7):1102-1111.

17. Abe T, Amano H, Kobayashi $\mathrm{T}$, et al. Preoperative neutrophil-to-lymphocyte ratio as a prognosticator in early stage pancreatic ductal adenocarcinoma. Eur J Surg Oncol. 2018;44(10):1573-1579.

18. Conroy T, Hammel P, Hebbar M, Ben Abdelghani M, Wei AC, Raoul JL, et al. FOLFIRINOX or Gemcitabine as Adjuvant Therapy for Pancreatic Cancer. N Engl J Med. 2018;379:2395-406.

19. Kleeff J, Korc M, Apte M, et al. Pancreatic cancer. Nat Rev Dis Primers. 2016;2:16022.

20. Makino I, Kitagawa $\mathrm{H}$, Ohta $\mathrm{T}$, et al. Nerve plexus invasion in pancreatic cancer: spread patterns on histopathologic and embryological analyses. Pancreas. 2008;37(4):358-365.

21. Kitagawa H, Ohta T, Makino I, et al. Carcinomas of the ventral and dorsal pancreas exhibit different patterns of lymphatic spread. Front Biosci. 2008;13:2728-2735.

\section{Tables}

Table 1. Demographic and Pathologic Findings Between Two Groups 
Standard $(n=79)$

Extended (74)

$\mathrm{P}$

Clinical variables

\begin{tabular}{|c|c|c|c|}
\hline Age, mean (SD), y & $59.48(10.55)$ & $57.20(9.91)$ & 0.172 \\
\hline Sex, M:F & $1.55: 1$ & $1.55: 1$ & 0.995 \\
\hline Initial CEA, median (IQR), ng/ml & $3.79(2.06-6.46)$ & $3.36(1.98-5.16)$ & 0.537 \\
\hline Initial CA125, median (IQR), U/ml & $17.74(13.45-25.39)$ & $18.00(12.73-38.18)$ & 0.774 \\
\hline Initial CA19-9, median (IQR), U/ml & $\begin{array}{l}198.60(49.22- \\
582.20)\end{array}$ & $\begin{array}{l}170.35(45.56- \\
833.50)\end{array}$ & 0.923 \\
\hline Initial albumin, median (IQR), g/L & $39.40(36.9-41.8)$ & $39.65(36.18-43.05)$ & 0.638 \\
\hline Operation time, median (IQR), min & $324(270-390)$ & $350(295-430)$ & 0.038 \\
\hline Transfusion (RBC+FFP), quantity (\%) & & & 0.289 \\
\hline $0 \mathrm{ml}$ & $58(73.43)$ & $48(64.87)$ & \\
\hline $0-400 \mathrm{ml}$ & $6(7.59)$ & $9(12.16)$ & \\
\hline $400-800 \mathrm{ml}$ & $6(7.59)$ & $10(13.51)$ & \\
\hline$>800 \mathrm{ml}$ & $9(11.39)$ & $7(9.46)$ & \\
\hline Infusion, median (IQR), ml & $3700(3000-4400)$ & $4100(3375-4725)$ & 0.047 \\
\hline EBL, median (IQR), ml & $300(200-600)$ & $425(300-725)$ & 0.064 \\
\hline Follow-up, median, $\mathrm{m}$ & 30.27 & 27.55 & \\
\hline Portal vein resection, quantity (\%) & 27邓34.17囚 & $22 \llbracket 29.72 \rrbracket$ & 0.556 \\
\hline
\end{tabular}

Pathologic variables

R1 resection, quantity (\%)

12(15.19)

10(13.51)

0.768

Tumor size, median (IQR), cm

3.1(2.5-4.0)

$3.0(2.5-4.0)$

0.870

T stage, quantity (\%)

0.790

T1

6(7.6)

$5(6.8)$

$\mathrm{T} 2$

58(73.4)

57(77.0)

T3

15(19.0)

12(16.2)

Total retrieved lymph nodes, median (IQR)

18(16-19)

24(22-26)

$₫ 0.001$

Positive lymph nodes, quantity (\%)

0.209 


\begin{tabular}{|lll|}
\hline 0 & $36(45.57)$ & $32(43.24)$ \\
\hline $3-3$ & $16(20.25)$ & $8(10.81)$ \\
\hline$>6$ & $25(31.65)$ & $30(40.54)$ \\
\hline AJCC stage (8th edition) & $2(2.53)$ & $4(5.41)$ \\
\hline IA & & \\
\hline IB & $3(3.8)$ & $3(4.1)$ \\
\hline IIA & $29(36.7)$ & $23(31.1)$ \\
\hline IIB & $4(5.1)$ & $6(8.1)$ \\
\hline III & $29(36.7)$ & $30(40.5)$ \\
\hline $\begin{array}{l}\text { AJCC indicates American Joint Committee on Cancer; CEA, carcinoembryonic antigen; CA125, cancer } \\
\text { antigen 125; CA19-9, cancer antigen 19-9; RBC, red blood cell; FFP, fresh frozen plasma; EBL, } \\
\text { estimated blood loss }\end{array}$ & $12(16.2)$ \\
\hline
\end{tabular}

\section{Table 2. Morbidity and Mortality Between Standard Group and Extended Group}




\begin{tabular}{|c|c|c|c|}
\hline & Standard $(n=79)$ & Extended $(n=74)$ & $P$ \\
\hline Postoperative hospital stay, mean (SD), d & 14.66(10.32) & $12.65(4.93)$ & $0.131^{\mathrm{e}}$ \\
\hline In-hospital death, quantity (\%) & $3(3.8)$ & $2(2.7)$ & NS \\
\hline Complications, quantity (\%) & $31(39.24)$ & $33(44.59)$ & $0.502^{f}$ \\
\hline Pancreatic fistula ${ }^{a}$ & $8(10.12)$ & $12(16.21)$ & \\
\hline Delayed gastric emptying (DGE) $b$ & $8(10.12)$ & $9(12.16)$ & \\
\hline Diarrhea $^{\mathrm{c}}$ & $7(8.86)$ & $9(12.16)$ & \\
\hline Intra-abdominal bleeding & $6(7.59)$ & $4(5.40)$ & \\
\hline Gastrointestinal bleeding & $4(5.06)$ & $3(4.05)$ & \\
\hline Severe sepsis & $3(3.80)$ & $2(2.70)$ & \\
\hline Pulmonary infection & $9(11.39)$ & $8(10.81)$ & \\
\hline Re-operation & $6(7.59)$ & $4(5.40)$ & \\
\hline Others ${ }^{d}$ & $7(8.86)$ & $10(13.51)$ & \\
\hline \multicolumn{4}{|c|}{$\begin{array}{l}\text { a Only grade B and C pancreatic fistula was counted, }{ }^{\mathrm{b}} \text { Only grade B and C DGE was counted, }{ }^{\mathrm{c}} \\
\text { Postoperative day } 3 \text { mouths, }{ }^{\mathrm{d}} \text { Including chylous fistula, wound infection, adhesive intestinal } \\
\text { obstruction, }{ }^{\mathrm{e}} \text { Student t test, }{ }^{\mathrm{f}} \text { Chi-squared test, NS indicates not significant }\end{array}$} \\
\hline
\end{tabular}

Table 3. Univariate and Multivariate Analyses of Survival 


\begin{tabular}{|c|c|c|c|c|c|c|}
\hline & \multirow[b]{2}{*}{$\mathrm{N}$} & \multirow[b]{2}{*}{ 2YSRヌ\% } & \multirow{2}{*}{$\begin{array}{l}\text { Univariate } \\
\mathrm{P}\end{array}$} & \multicolumn{3}{|c|}{ Multivariate } \\
\hline & & & & $\mathrm{HR}$ & $\begin{array}{l}95 \% \\
\mathrm{Cl}\end{array}$ & $P$ \\
\hline Sex, M/F & $88 / 58$ & $37.5 / 25.8$ & 0.076 & & & \\
\hline Age, $\geq 65 / \bowtie 65$ & $43 / 103$ & $38.8 / 30.1$ & 0.918 & & & \\
\hline Initial CEA囚 $\geq 5.0 / \otimes 5.0$ & $49 / 97$ & $37.1 / 30.6$ & 0.610 & & & \\
\hline 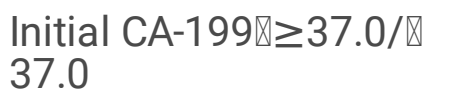 & $114 / 32$ & $36.7 / 19.9$ & 0.258 & & & \\
\hline $\begin{array}{l}\text { OP extent, } \\
\text { standard/extended }\end{array}$ & $75 / 71$ & $39.5 / 25.3$ & 0.034 & 1.67 & $\begin{array}{l}1.130- \\
2.254\end{array}$ & 0.010 \\
\hline $\begin{array}{l}\text { Portal vein resection, } \\
\text { Yes/No }\end{array}$ & $44 / 102$ & $29.7 / 34.2$ & 0.749 & & & \\
\hline R state R0/R1 & $125 / 21$ & $35.3 / 17$ & 0.428 & & & \\
\hline T stage $\mathrm{T} 1 / \mathrm{T} 2 / \mathrm{T} 3$ & $8 / 112 / 26$ & $37.5 / 32.4 / 32.7$ & 0.937 & & & \\
\hline $\mathrm{N}$ stage $\mathrm{N} 0 / \mathrm{N}+$ & $62 / 84$ & $39.5 / 28$ & 0.044 & 1.254 & $\begin{array}{l}0.823- \\
1.910\end{array}$ & 0.292 \\
\hline Stage I/II/III & $53 / 68 / 25$ & $44.6 / 34 / 8$ & 0.001 & 1.338 & $\begin{array}{l}0.992- \\
1.804\end{array}$ & 0.056 \\
\hline Histology, WD/MD/PD & $5 / 116 / 25$ & $75 / 36.6 / 5$ & 0.002 & 2.190 & $\begin{array}{l}1.370- \\
3.502\end{array}$ & 0.001 \\
\hline Adjuvant treatment, +/- & $61 / 85$ & $50.3 / 21.1$ & $<0.001$ & 2.226 & $\begin{array}{l}1.464- \\
3.384\end{array}$ & $<0.001$ \\
\hline Perineural invasion, +/- & $120 / 26$ & $32.3 / 35.2$ & 0.678 & & & \\
\hline $\begin{array}{l}\text { Endolymphatic tumor } \\
\text { emboli, +/- }\end{array}$ & $32 / 114$ & $16.4 / 37.8$ & 0.154 & & & \\
\hline $\begin{array}{l}\text { M: male, F: female, OP: } \\
\text { differentiated, }\end{array}$ & ion, WD: w & differentiated, $\mathrm{M}$ & : moderatel & differen & ated, $P[$ & oor \\
\hline
\end{tabular}

\section{Figures}


28 Excluded

11 Patient declined to participate

17 Did not meet inclusion criteria

212 Randomized

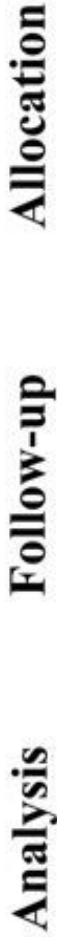

108 Allocated to Standard group

97 Received intervention as assigned

11 Did not receive assigned intervention

11 Unresectable or metastasis

\section{Excluded}

2 other pathology

4 rule violation

9 Lost to follow-up

7 lost contact

2 withdrawal from study participation

3 Excluded from analysis

3 inadequate case report form

79 Included in analysis
104 Allocated to Extended group

95 Received intervention as assigned

9 Did not receive assigned intervention

9 Unresectable or metastasis

\section{Excluded}

2 other pathology

7 rule violation

10 Lost to follow-up

9 lost contact

1 withdrawal from study participation

2 Excluded from analysis

$\mathbf{2}$ inadequate case report form

74 Included in analysis

Figure 1

Legend: Consort Diagram 

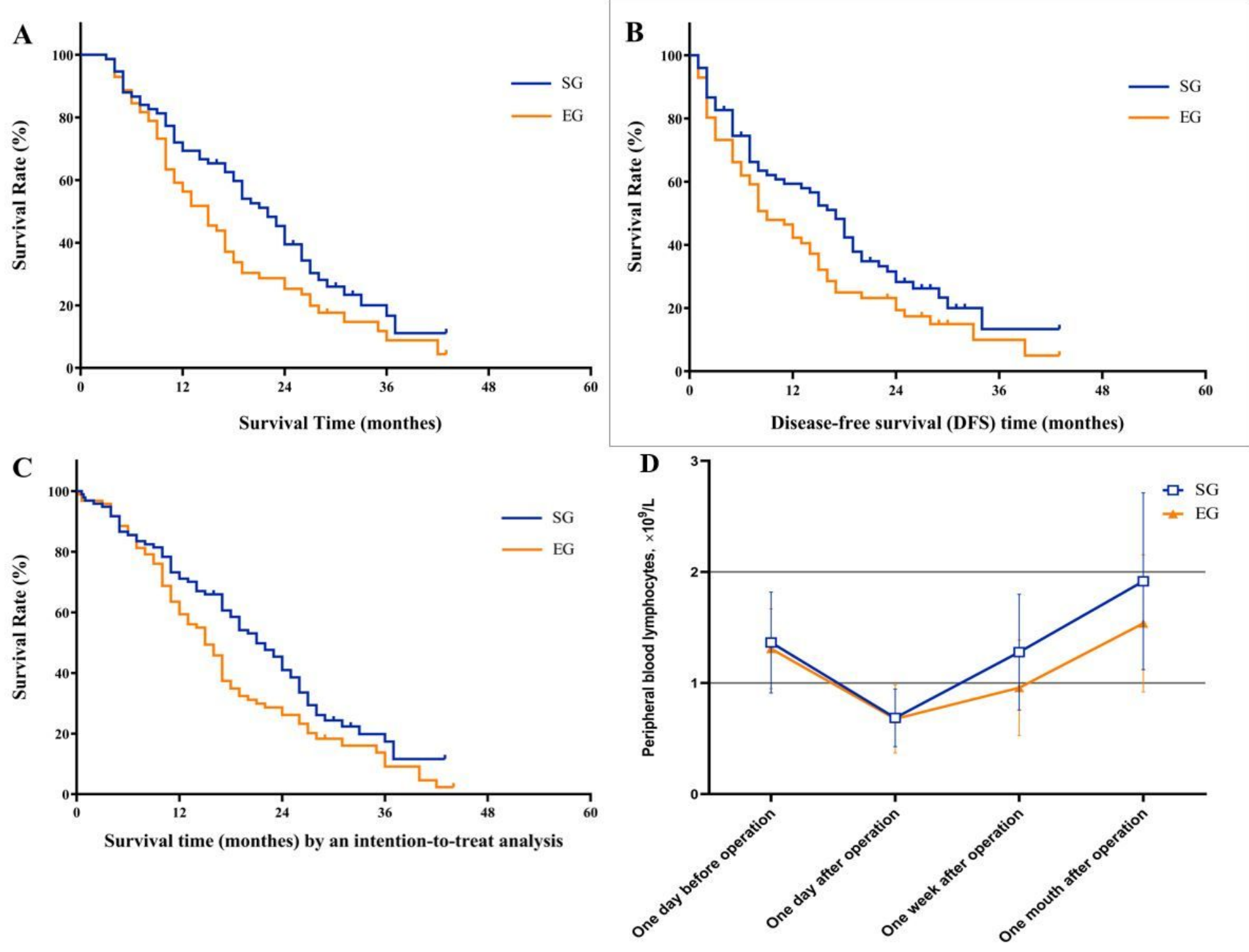

Figure 2

Legend: Survival curves between two groups. A: Overall survival in two groups, B: Disease-free survival (DFS) in two groups, C: Overall survival by an intention-to-treat analysis, D: The trend of peripheral blood lymphocytes between two groups in different time. SG: standard lymphadenectomy group, EG: extended lymphadenectomy group 

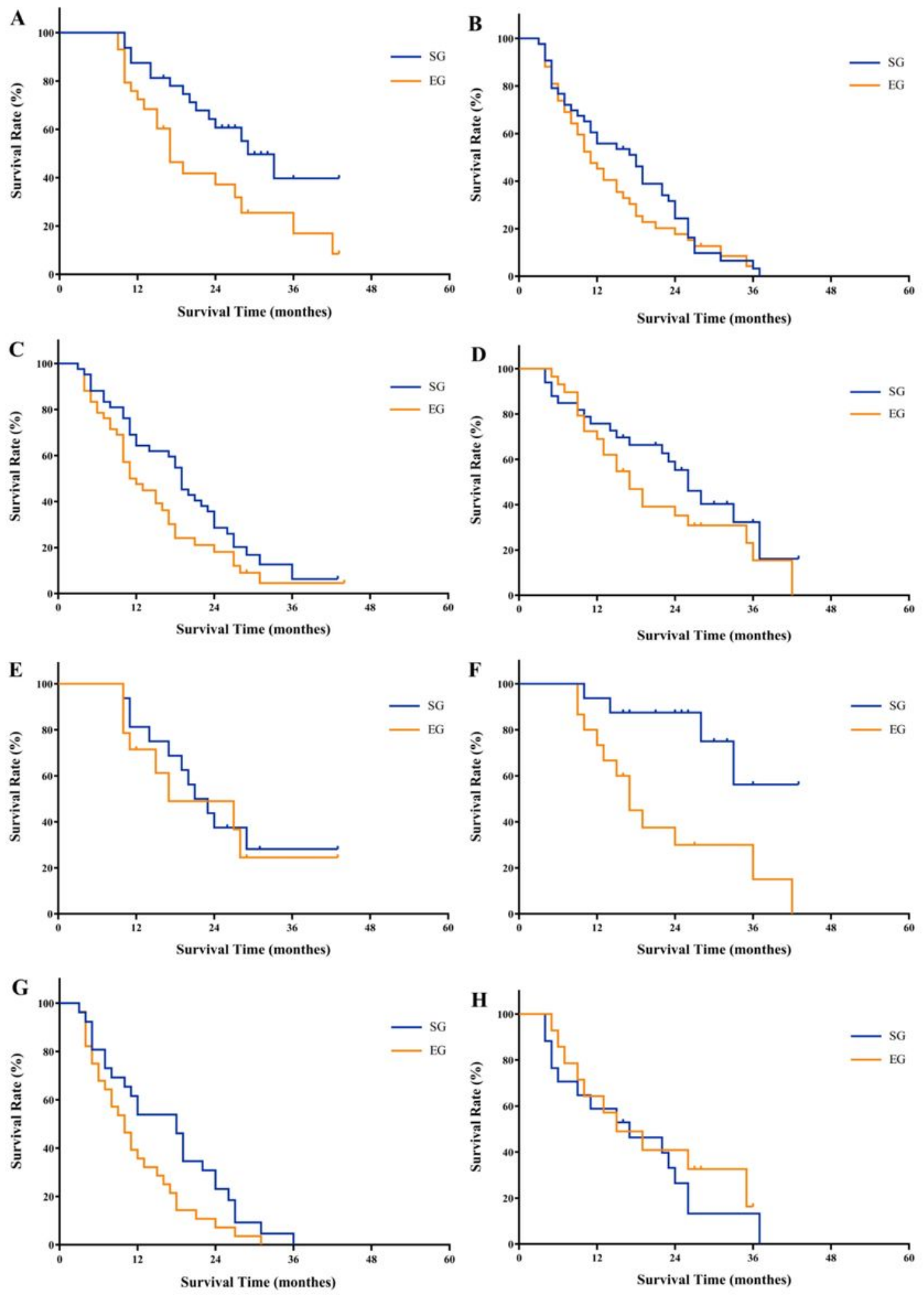

Figure 3

Legend: A: Survival curves for patients receiving chemotherapy in two groups, B: Survival curves for patients not receiving chemotherapy in two groups, C: Survival curves for patients with positive lymph nodes in two groups, D: Survival curves for patients with negative lymph nodes in two groups, E: Survival curves for patients with positive lymph nodes and receiving chemotherapy in two groups, F: Survival curves for patients with negative lymph nodes and receiving chemotherapy in two groups, G: Survival 
curves for patients with positive lymph nodes and not receiving chemotherapy in two groups, H: Survival curves for patients with negative lymph nodes and not receiving chemotherapy in two groups.SG: standard lymphadenectomy group, EG: extended lymphadenectomy group

\section{Supplementary Files}

This is a list of supplementary files associated with this preprint. Click to download.

- SupplementTable1.docx

- SupplementFigure1and2.docx

- CONSORTChecklist.doc 